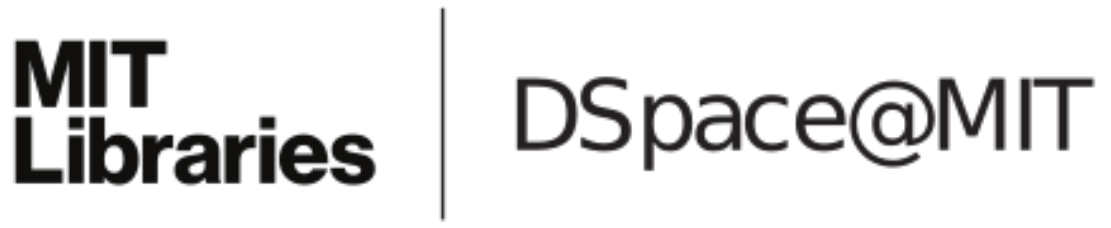

\author{
MIT Open Access Articles \\ Doublon-Hole Correlations and Fluctuation \\ Thermometry in a Fermi-Hubbard Gas
}

The MIT Faculty has made this article openly available. Please share how this access benefits you. Your story matters.

As Published: 10.1103/PHYSREVLETT.125.113601

Publisher: American Physical Society (APS)

Persistent URL: https://hdl.handle.net/1721.1/135292

Version: Final published version: final published article, as it appeared in a journal, conference proceedings, or other formally published context

Terms of Use: Article is made available in accordance with the publisher's policy and may be subject to US copyright law. Please refer to the publisher's site for terms of use. 


\title{
Doublon-Hole Correlations and Fluctuation Thermometry in a Fermi-Hubbard Gas
}

\author{
Thomas Hartke $\odot$, Botond Oreg $\odot$, Ningyuan Jia, and Martin Zwierlein๑ \\ Department of Physics, MIT-Harvard Center for Ultracold Atoms, and Research Laboratory of Electronics, \\ Massachusetts Institute of Technology, Cambridge, Massachusetts 02139, USA
}

(Received 26 March 2020; revised 19 July 2020; accepted 14 August 2020; published 9 September 2020)

\begin{abstract}
We report on the single atom and single site-resolved detection of the total density in a cold atom realization of the 2D Fermi-Hubbard model. Fluorescence imaging of doublons is achieved by splitting each lattice site into a double well, thereby separating atom pairs. Full density readout yields a direct measurement of the equation of state, including direct thermometry via the fluctuation-dissipation theorem. Site-resolved density correlations reveal the Pauli hole at low filling, and strong doublon-hole correlations near half filling. These are shown to account for the difference between local and nonlocal density fluctuations in the Mott insulator. Our technique enables the study of atom-resolved charge transport in the Fermi-Hubbard model, the site-resolved observation of molecules, and the creation of bilayer FermiHubbard systems.
\end{abstract}

DOI: $10.1103 /$ PhysRevLett.125.113601

Understanding strongly correlated quantum systems poses a major challenge both for theory and experiment. Recent years have seen a significant progress in simulating quantum many-body physics with ultracold atoms [1-4]. In particular, the Fermi-Hubbard model plays a paradigmatic role in the study of strongly correlated fermions, most prominently for understanding high- $T_{c}$ superconductivity [5]. Quantum gas microscopes $[4,6,7]$ of fermionic atoms [8-12] provide the ability to explore fermion correlations with single-atom, single-site resolution. Recent works have demonstrated the metal and Mott insulator crossover [13-16], studied spin and charge correlations [17-22], revealed magnetic polarons [23] and studied spin [24], charge [25], and heat transport [26]. However, most experiments employ fluorescence imaging directly on the lattice used for Hubbard physics. Light-assisted collisions then remove atom pairs residing on the same lattice site from the image $[27,28]$, leading to parity projection $[6,7]$ and, in particular, the appearance of doubly occupied sites (doublons) as holes. Such Fermi gas microscopes thus measure only the density of singly occupied sites (singlons), i.e., the local moment [19]. The full density can be obtained via absorption imaging [13] but without single site resolution, or by selectively imaging either singlons or doublons [29].

Revealing the microscopic correlations giving rise to macroscopic observables of the Fermi-Hubbard model requires single-shot measurements of the full density. As the prime example, the fluctuation-dissipation theorem [30] relates the compressibility to the global number fluctuations of the system via the temperature, requiring measurements of the total density sensitive to atomic shot noise [31-33]. The importance of nonlocal density fluctuations has been demonstrated $[16,34]$, but revealing their microscopic origin requires site-resolved density measurements.
Progress in fluorescence imaging of the total density was achieved with superlattices [18] that spatially separated atom pairs into distinct wells, revealing the interplay of charge and spin [23] in systems of $\sim 6 \times 6$ sites.

In this Letter, we introduce a bilayer Fermi gas microscope enabling full site-resolved density readout of large ( 1500 sites) 2D Fermi-Hubbard systems in a single fluorescence image. This directly yields the equation of state as pressure, compressibility, and doublon density are obtained as a function of density. Site-resolved density correlations reveal the importance of nonlocal correlations, from the Pauli hole at low filling to strong doublonhole correlations at half filling. The measured density fluctuation and compressibility directly yield a theoryindependent thermometer via the fluctuation-dissipation theorem [30]. In the Mott insulator, we find strongly correlated nearest-neighbor doublon-hole pairs, required to compensate for local density fluctuations to yield the near-vanishing compressibility.

To record the full density information, our setup consists of a bilayer optical lattice potential beneath a microscope objective, shown schematically in Fig. 1(a). In the experiment, a 2D Fermi-Hubbard gas is prepared in a single horizontal layer of a 3D optical lattice as reported in Ref. [8], with horizontal (vertical) lattice spacing of $a=$ $541 \mathrm{~nm}(3 \mu \mathrm{m})$. For imaging, the depth of the horizontal lattices is increased to prevent tunneling in the 2D plane. Some lattice sites will contain doublons. We now impose a vertical superlattice [purple arrow in Fig. 1(a)] with $532 \mathrm{~nm}$ spacing, created by retroreflecting a $1064 \mathrm{~nm}$ laser beam off the flat surface of the hemispheric microscope objective. Driven by Feshbach enhanced repulsive interactions, two atoms originally in a single lattice site separate vertically into different wells [Fig. 1(b)] [35]. After the splitting 

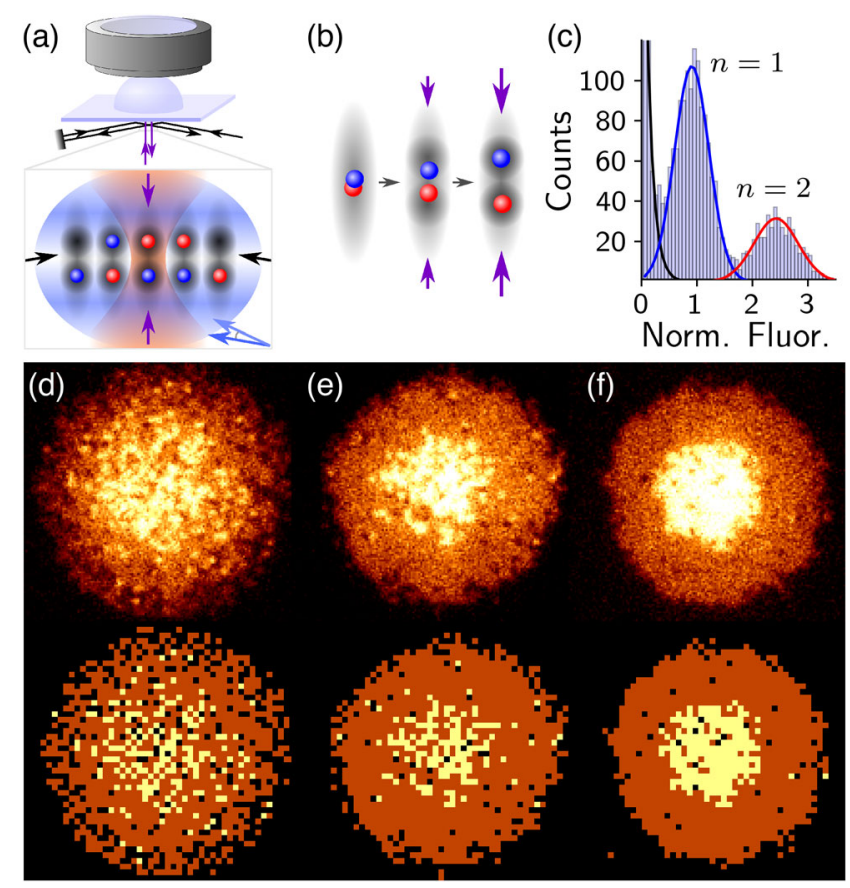

FIG. 1. Observing the formation of fermionic Mott and band insulators via total density readout in a bilayer microscope. (a) A degenerate Fermi gas is prepared in a 2D optical lattice potential (black arrows) beneath a microscope objective. A vertical superlattice (purple arrows, $532 \mathrm{~nm}$ separation) can hold two atoms in different layers simultaneously within the microscope focus (collecting $770 \mathrm{~nm}$ light, orange shading). The intensity of Raman light (blue arrow and shading) used for imaging is tunable for each layer by changing the beam angle. (b) Repulsively interacting atom pairs, originally in a single well, are split by imposing the vertical superlattice before imaging. (c) A typical fluorescence histogram, clearly indicating the presence of $n=1$ and $n=2$ atoms per lattice site. (d)-(f) A strongly correlated metal $(U / t \sim 7)$ turns into a fermionic Mott insulator (with $n=1$ ) surrounding a band insulator $(n=2)$ upon increasing $U / t$ to (e) 19 and (f) 84 . Reconstructed lattice occupations shown below.

process, Raman sideband cooling is performed as in Ref. [8] and emitted optical pumping photons are collected through the microscope objective. In contrast to previous work with bosons [36], the layer separation is within the depth of focus of the microscope, allowing atoms in both layers to be simultaneously imaged onto the same diffraction limited spot on the camera.

We now demonstrate that separated atoms continue to fluoresce without light-induced loss. By raising the harmonic trapping potential, we create a band insulator at the center of the cloud and perform the vertical separation of atom pairs before imaging. Figure 1(c) shows a typical histogram of an image, with fluorescence counts from singly occupied sites clearly distinguishable from those for originally doubly occupied sites. The fluorescence obtained from atoms in each layer can be tuned via the intensity of Raman light [35]. Typical images are shown in

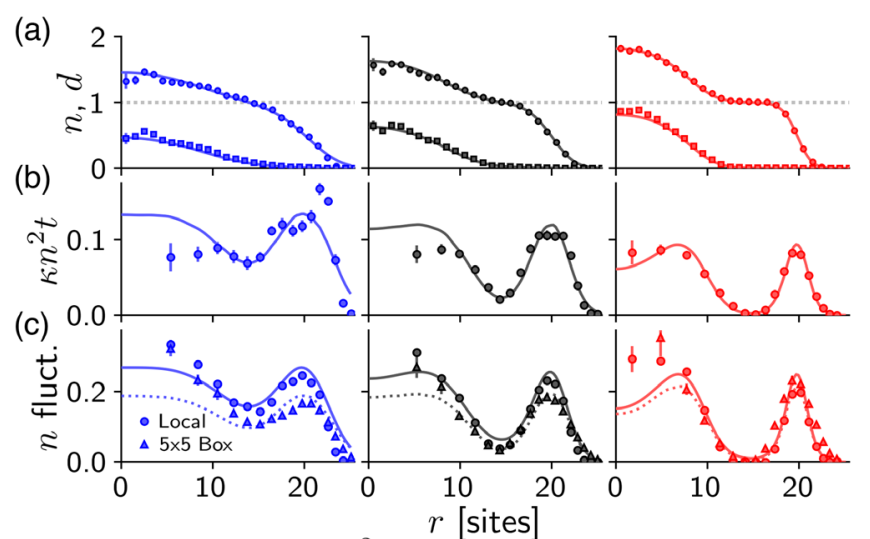

(d) $P$

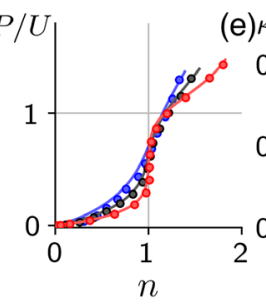

e) $\kappa n^{2} t$
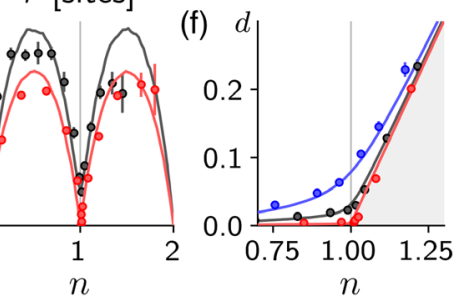

FIG. 2. Equation of state of the 2D Fermi-Hubbard model. (a) Radially averaged profiles of total density (circles) and doublon density (squares) in a Fermi-Hubbard gas at $U / t=$ 7.1(4) (blue), 11.8(5) (black), and 25.3(6) (red). (b) Measured normalized compressibility $\kappa n^{2} t$. (c) Local density fluctuations $\left\langle\hat{n}^{2}\right\rangle-\langle\hat{n}\rangle^{2}$ (circles) and total atom number fluctuations per area $\left(\left\langle\hat{N}^{2}\right\rangle-\langle\hat{N}\rangle^{2}\right) /$ area in a $5 \times 5$ box (triangles). (d)-(f) Thermodynamic variables vs density: (d) normalized pressure $P / U$, (e) compressibility $\kappa n^{2} t$, and (f) doublon density $d$. All lines show Monte Carlo predictions [40] for $T / t=1.4$ (blue), $T / t=1.6$ (black), and $T / t=2.25$ (red) with the same $U / t$ as the data. Here and elsewhere, data are corrected by measured rates of loss $(\sim 5 \%)$ and hopping ( 5\%) [35].

Figs. 1(d)-1(f), for various values of the ratio $U / t$ between the on-site interaction strength $U$, and the tunneling rate $t$ [15]. Figure $1(\mathrm{~d})$ shows a strongly correlated metal at $U / t \sim 7$. As $U / t$ is increased, the tell-tale "wedding cake" structure emerges, with a central band insulator at a fluorescence level corresponding to $n=2$ surrounded by a Mott insulator at lower fluorescence corresponding to $n=1$. Singly and doubly occupied sites are clearly distinguished [35], leading to the digitized images below.

Figure 2(a) shows examples of radially averaged density $n$ (circles) and doublon density $d$ (squares) at varying $U / t$. On a given lattice site, we set $d=1$ when $n=2$, and the hole density $h=1$ when $n=0$. With increasing repulsion (from left to right) a Mott plateau emerges at $n=1$. The compressibility $\kappa$ in Fig. 2(b) is obtained via the local density approximation from the variation in the measured local potential $V(r)$ as $\kappa n^{2}=\partial n /\left.\partial \mu\right|_{T}=-\partial n /\left.\partial V\right|_{T}[37,38]$. It is observed to vanish in the region of the Mott plateau, directly indicating insulating behavior $[13,39]$. A simultaneous reduction in local (on-site) fluctuations in the density in Fig. 2(c), $\left\langle\hat{n}^{2}\right\rangle-\langle\hat{n}\rangle^{2}=n(1-n)+2 d$, is caused by the reduced double occupancy $d$ in the Mott insulator at $n=1$. 
Access to the total density directly yields a measurement of the equation of state of the Fermi-Hubbard model. The canonical equation of state relates pressure $P=$ $P(n, T, U, t)$ to density, temperature $T$, and interaction parameters $U$ and $t$. However, one is free to replace, e.g., temperature by any other thermodynamic variable like the doublon fraction, and, e.g., $t$ by compressibility $\kappa$, thereby obtaining an equation of state of directly and locally observable quantities $[37,38]$. From the variation of density with potential $n(V)$ one obtains the pressure $P(V)=$ $\int_{-\infty}^{\mu_{0}-V} n\left(\mu^{\prime}\right) d \mu^{\prime}=\int_{V}^{\infty} n\left(V^{\prime}\right) d V^{\prime} \quad$ [34,37,41-43]. Together with $P$, one has the compressibility $\kappa n^{2}=n \partial n /\left.\partial P\right|_{T}$, and the dimensionless doublon fraction $d$, all as a function of density $n$ [Figs. 2(d)-2(f), respectively]. For the strongest interactions it can be observed how the pressure needs to rise above $U$ before breakdown of the Mott insulator occurs and the density can grow above $n=1$. Finally, the compressibility, together with the total density fluctuations in Fig. 2(c) directly yield the temperature $T$ via the fluctuation-dissipation theorem. To this end, in the following we will investigate density correlations.

The density correlations of a noninteracting Fermi gas are determined by Pauli exclusion, which forbids two identical fermions to share the same phase-space cell. At nondegenerate temperatures, the probability to find two like fermions near each other is suppressed for distances smaller than the thermal de Broglie wavelength $\lambda_{\mathrm{dB}} \sim a \sqrt{t / T}$. As the phase space density $n \lambda_{\mathrm{dB}}^{2} / a^{2} \gtrsim 1$, i.e., $T \lesssim n t$, the size of this Pauli exclusion hole saturates to the spacing $a / \sqrt{n}$ between identical fermions. In a two-state mixture of fermions and at low filling, repulsion between unlike spins further deepens the correlation hole between particles. These nonlocal anticorrelations have the effect of reducing the total atom number fluctuations in a given region. Any local upward density fluctuation will be partially compensated by a reduction in nearby density. In Fig. 2(c) we demonstrate that density fluctuations are reduced in a $5 \times 5$ site box (triangles) compared to onsite fluctuations (circles), indicating the presence of nonlocal anticorrelations between fermions.

We now use the full site-resolved density readout of our microscope to directly measure the correlation hole in an interacting Fermi-Hubbard lattice gas. The Pauli hole has been inferred from antibunching of the parity-projected density in previous work [19]. The connected densitydensity correlation $\left\langle\hat{n}_{i} \hat{n}_{i+\delta}\right\rangle_{C}=\left\langle\hat{n}_{i} \hat{n}_{i+\delta}\right\rangle-n_{i} n_{i+\delta}$ characterizes the nontrivial correlation of finding two particles a distance of $\delta$ lattice sites apart, beyond that for uncorrelated particles at the same density. Figure 3(a) shows the spatial dependence of $\left\langle\hat{n}_{i} \hat{n}_{i+\delta}\right\rangle_{C}$ at various densities. Strong nonlocal anticorrelations are clearly visible. Figure 3(b) reports the total, local, and nonlocal density fluctuations. Significant negative nonlocal correlations indicate a de Broglie wavelength that extends over multiple lattice sites, requiring $T \sim t[44,45]$. We note that nonlocal correlations
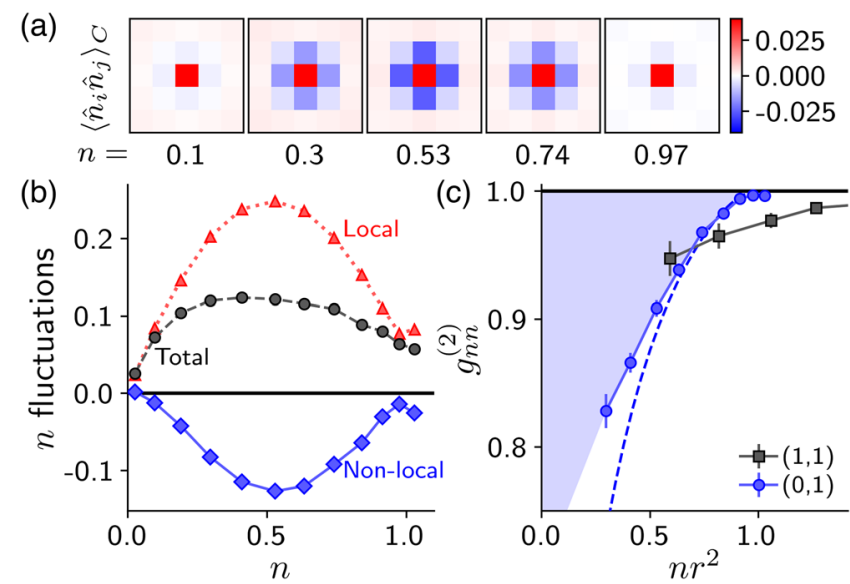

FIG. 3. Measurement of nonlocal density correlations in the 2D Fermi-Hubbard model. (a) Connected density-density correlations at various densities at $U / t=11.8(5)$. (b) Density fluctuations $\sum_{\delta}\left\langle\hat{n}_{i} \hat{n}_{i+\delta}\right\rangle_{C}$ (total, black circles), $\left\langle\hat{n}^{2}\right\rangle-\langle\hat{n}\rangle^{2}$ (local, red triangles), and $\sum_{\delta \neq 0}\left\langle\hat{n}_{i} \hat{n}_{i+\delta}\right\rangle_{C}$ (nonlocal, blue diamonds). (c) Density-density correlation function $g_{n n}^{(2)}$ for displacements $(0,1)$ (blue circles) and $(1,1)$ (black squares) vs $n r^{2}$, and theory for a noninteracting single-component Fermi lattice gas for displacement $(0,1)$ (blue dashed line) at $T / t=0.69$ (consistent with thermometry in Fig. 4). The shading is a guide to the eye, indicating the correlation hole due to Pauli exclusion between like spins and repulsion between unlike spins.

were inferred but not directly measured in Ref. [16]. The magnitude of local and nonlocal fluctuations is maximal at $n \approx 0.5$, a direct consequence of strong on-site repulsion between unlike spins. This effectively reduces the available area for each species by half. On-site density fluctuations are thus equal to that of a single spin species in half the area, of density $n$ and binomial fluctuation $\left\langle\hat{n}^{2}\right\rangle-\langle\hat{n}\rangle^{2} \approx$ $n(1-n)$, peaking at $n=0.5$. Pauli exclusion requires a corresponding anticorrelation in the area surrounding a given local fluctuation, so nonlocal fluctuations peak near the same filling.

The spatial Pauli hole is directly visualized through the density-density correlation function $g_{n n}^{(2)}=\left\langle\hat{n}_{i} \hat{n}_{i+\delta}\right\rangle / n_{i} n_{i+\delta}$. Figure 3(c) shows the measured $g_{n n}^{(2)}$ for nearest-neighbor and next-nearest neighbor displacements $\delta$ versus $n r^{2}$, which normalizes distance by the Fermi wavelength. The strong reduction of $g_{n n}^{(2)}$ within one interparticle spacing (blue shaded region) represents the direct observation of the correlation hole due to Pauli exclusion of like spins, and repulsion of unlike spins. The $g_{n n}^{(2)}$ for a single, noninteracting fermionic species at the full density $n$ shows good agreement, highlighting again that strong interspin repulsion reduces the available area for a given spin species by half.

With access to both the measured microscopic density fluctuations (Fig. 3) and the macroscopic compressibility (Fig. 2), we are now in the position to probe the fundamental correspondence between fluctuations and response 


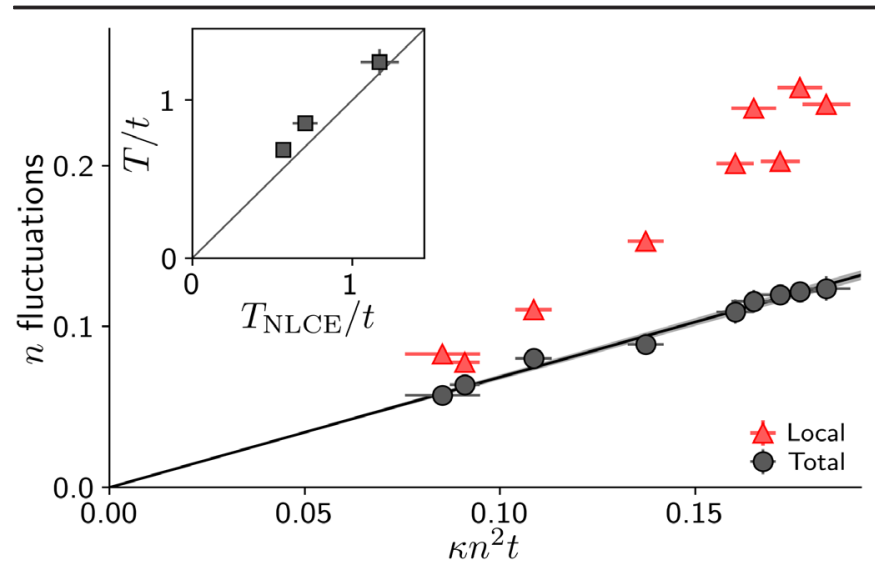

FIG. 4. Direct thermometry via density-density correlations. Density fluctuations vs normalized compressibility $\kappa n^{2} t$ for $U / t=11.8(5)$ : local fluctuations $\left\langle\hat{n}^{2}\right\rangle-\langle\hat{n}\rangle^{2}$ (red triangles) and total fluctuations $\sum_{\delta}\left\langle\hat{n}_{i} \hat{n}_{i+\delta}\right\rangle_{C}$ (black circles). A linear fit of total fluctuations vs compressibility, fixed through the origin (black solid line), provides the temperature $T=$ $\sum_{\delta}\left\langle\hat{n}_{i} \hat{n}_{i+\delta}\right\rangle_{C} / n^{2} \kappa$ (gray shading shows statistical uncertainty). Inset: Measured temperatures vs inferred temperatures $T_{\mathrm{NLCE}}$ from theoretical fits to radial singlon profiles, after heating the system for variable time [35]. Errors are only statistical.

in thermal quantum systems [46]. The general density fluctuation-dissipation theorem

$$
\kappa n^{2}=\left.\frac{\partial n_{i}}{\partial \mu}\right|_{T}=\beta \sum_{\delta}\left\langle\hat{n}_{i} \hat{n}_{i+\delta}\right\rangle_{C},
$$

where $\beta=1 / T$ relates directly measurable macroscopic and microscopic quantities without reference to any theoretical model [30]. Significantly, nonlocal density correlations will remain a sensitive thermometer down to $T=0$ for any compressible system because $\kappa n^{2}=$ $\partial n_{i} /\left.\partial \mu\right|_{T}$ will saturate to the density of states at low temperatures [32]. For example, in low density metallic regions with a free particle energy dispersion, $\partial \mu /\left.\partial n_{i}\right|_{T} \rightarrow$ $2 \pi t$ as $T \rightarrow 0$, which implies sensitivity to temperatures $T \ll t$. Moreover, by averaging over the system's area, Eq. (1) relates compressibility to the global atom number fluctuations: $\kappa n^{2}=\beta\left(\left\langle\hat{N}^{2}\right\rangle-\langle\hat{N}\rangle^{2}\right) /$ area. In small subsystems, however, number fluctuations are enhanced due to nonlocal correlations across boundaries. This is the origin of the violation of the area law for entanglement entropy already present for noninteracting fermions [47-49].

Figure 4 shows the total connected density-density correlation (black circles) versus the normalized compressiblity $\kappa n^{2} t$ for the same dataset as Fig. 3. A linear fit results in a temperature of the cloud of $T / t=0.69(2)$ using Eq. (1). The entire inhomogeneous atomic gas contributes data, providing high statistical precision for this single parameter fit. Moreover, the agreement of the data with a linear fit demonstrates that any individual measurement realizes a spatially localized thermometer. For comparison, local fluctuations (red triangles) are nonlinear and are consistently larger than total fluctuations, highlighting again the importance of negative nonlocal correlations, inferred in Ref. [16].

We quantitatively benchmark the fluctuation thermometer by independently obtaining the temperature from fits of the radial singlon profiles of the same data to numerical linked-cluster expansion (NLCE) calculations [50]. Thermometry is repeated for different amounts of heating of the atom cloud $[19,35]$. As demonstrated in the inset of Fig. 4, the temperatures measured via fluctuation thermometry and those obtained from fits to NLCE agree. Note that in general, comparison of measured quantities to theory requires fitting to nonlinear and in some cases nonmonotonic functions, leading to difficulties in assessing systematic errors. In contrast, the sole sources of systematic uncertainty in fluctuation thermometry are the calibration of the trap potential, entering linearly into uncertainty in $T$, and measurable errors in the density. We have thus established a theory-independent, precise, and sensitive thermometer for interacting lattice fermions. The method is also ideally suited for homogeneous systems in box potentials [24], where density fluctuations in the presence of a well-calibrated linear gradient will provide access to the local temperature. This opens up prospects for the study of heat transport in the Fermi-Hubbard model.

The fluctuation-dissipation theorem provides insight into charge fluctuations in the Mott insulator at half-filling, at temperatures $T \ll U$, where the compressibility vanishes. In any system where either $T \rightarrow 0$ or $\kappa n^{2} \rightarrow 0$, Eq. (1) implies that local and nonlocal density fluctuations must cancel. For finite tunneling $t \sim T \ll U$, the system remains insulating, although the local operator $t$ acts as a perturbation that causes charge fluctuations over short distances [34]. The dominant contributions to $\left\langle\hat{n}_{i} \hat{n}_{j}\right\rangle_{C}=\left\langle\hat{d}_{i} \hat{d}_{j}\right\rangle_{C}+$ $\left\langle\hat{h}_{i} \hat{h}_{j}\right\rangle_{C}-2\left\langle\hat{d}_{i} \hat{h}_{j}\right\rangle_{C}$ are nearest neighbor doublon-hole fluctuations that occur with probability $\sim(t / U)^{2}$ [51]. Their existence has been inferred in Ref. [19] by observing bunching of holes after parity projection. For fermions, these nearest neighbor doublon-hole correlations signal spin singlet formation, as Pauli exclusion prevents tunneling for spin triplets.

Armed with full density readout, in Fig. 5 we now directly detect these doublon-hole fluctuations. At our temperatures $T \ll U$, where thermal fluctuations are frozen out, doublon-hole fluctuations are purely quantum in origin. Many of these isolated doublon-hole pairs can be directly observed as fluctuations within the strongly coupled Mott insulator in Fig. 1(e) $[U / t=18.8(5)]$. In Figs. 5(a)-5(c) we show the spatial dependence of the connected doublon-hole correlator $\left\langle\hat{d}_{i} \hat{h}_{j}\right\rangle_{C}$, the nearest neighbor correlator $\left\langle\hat{d}_{i} \hat{h}_{i+1}\right\rangle_{C}$, and the doublon-hole distribution function $g_{d h}^{(2)}=\left\langle\hat{d}_{i} \hat{h}_{i+1}\right\rangle / d_{i} h_{i+1}$ versus density at 


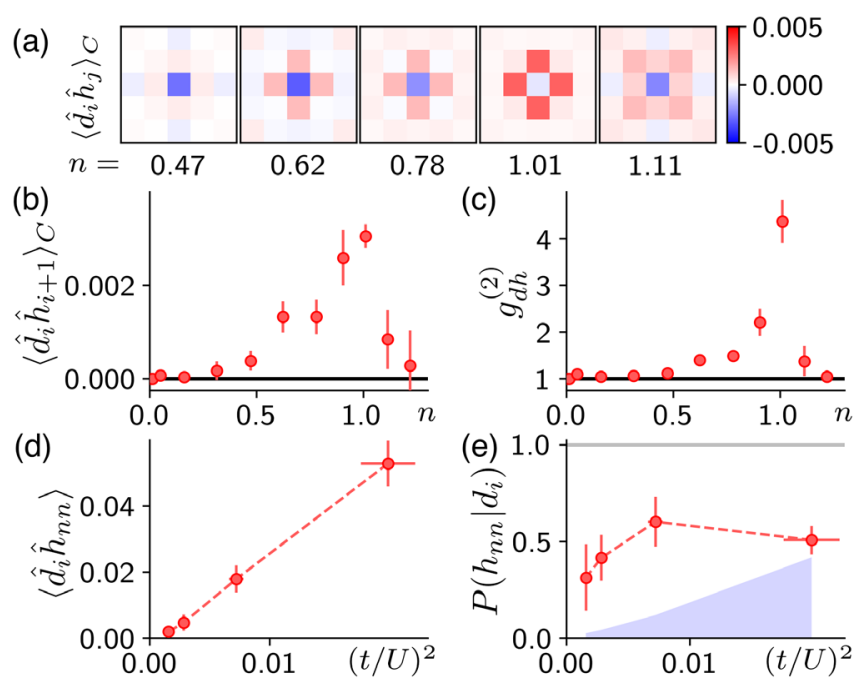

FIG. 5. Direct observation of doublon-hole correlations in the 2D Fermi-Hubbard model. (a) Spatial dependence of $\left\langle\hat{d}_{i} \hat{h}_{j}\right\rangle_{C}$ for $U / t=11.8(5)$ vs filling $n$. (b) Nearest neighbor correlations $\left\langle\hat{d}_{i} \hat{h}_{i+1}\right\rangle_{C}$ and (c) nearest neighbor $g_{d h}^{(2)}$ vs $n$. (d) Density of doublons with any nearest neighbor hole pair $\left\langle\hat{d}_{i} \hat{h}_{n n}\right\rangle=$ $\sum_{j \in n n}\left\langle\hat{d}_{i} \hat{h}_{j}\right\rangle$ at half filling vs $(t / U)^{2}$ [for $U / t=25.3(6), 18.8$ (5), 11.8(5), and 7.1(4)]. (e) Conditional probability of a nearest neighbor hole $P\left(h_{n n} \mid d_{i}\right)=\left\langle\hat{d}_{i} \hat{h}_{n n}\right\rangle / d_{i}$ at half filling compared to the expectation for a random distribution (blue shaded region) at the same hole and doublon density: $4 d$.

$U / t=11.8(5), \quad$ all of which demonstrate strongly enhanced local doublon-hole correlations near $n=1$.

In Fig. 5(d) we report the nearest neighbor doublon-hole pair density $\left\langle\hat{d}_{i} \hat{h}_{n n}\right\rangle=\sum_{j \in n n}\left\langle\hat{d}_{i} \hat{h}_{j}\right\rangle$ with respect to $(t / U)^{2}$. The linear relationship highlights the physical origin of doublon-hole pair correlations in a coherent, off-resonant tunneling process of amplitude $\sim t / U$. To demonstrate the strength of bunching, we obtain the conditional probability $P\left(h_{n n} \mid d_{i}\right)=\left\langle\hat{d}_{i} \hat{h}_{n n}\right\rangle / d_{i}$ to find a hole next to a doublon in Fig. 5(e). As a comparison, we also show the conditional probability for a Poisson process at the same hole and doublon density $4 d$ (blue shaded area). At small $t / U$, the conditional probability far exceeds random chance, showing that doublons and holes are tightly bound in a Mott insulator.

In conclusion, we demonstrate a robust method to measure the total site-resolved density in a cold-atom realization of the 2D Fermi-Hubbard model. We use this ability to directly detect nonlocal correlations, in particular the Pauli correlation hole at low filling and doublon-hole correlations in the Mott insulating region. Model-free thermometry is established via the fluctuationdissipation theorem. Using a magnetic field gradient, we can also perform spin dependent splitting [35], which will eventually allow simultaneous observation of both charge and spin. Our superlattice geometry opens up the ability to study bilayer and even multilayer
Fermi-Hubbard models, relevant for high-temperature superconductivity [52,53].

We would like to thank M. A. Nichols and H. Zhang for early contributions to the experiment, E. Kozik for stimulating discussions, and E. Khatami for providing NLCE calculations [19]. This work was supported by the NSF, ONR, an AFOSR MURI on Exotic Phases of Matter, the David and Lucile Packard Foundation, the Gordon and Betty Moore Foundation through Grant No. GBMF5279, and the Vannevar Bush Faculty Fellowship. M. Z. acknowledges support from the Alexander von Humboldt Foundation.

Note added.-After completion of our experimental work [54], a spin-resolved bilayer imaging technique was realized in Ref. [55].

[1] Ultracold Fermi Gases, edited by M. Inguscio, W. Ketterle, and C. Salomon (IOS Press, Amsterdam, 2008).

[2] I. Bloch, J. Dalibard, and W. Zwerger, Rev. Mod. Phys. 80, 885 (2008).

[3] The BCS-BEC Crossover and the Unitary Fermi Gas, edited by W. Zwerger (Springer, Berlin Heidelberg, 2012).

[4] C. Gross and I. Bloch, Science 357, 995 (2017).

[5] P. A. Lee, N. Nagaosa, and X.-G. Wen, Rev. Mod. Phys. 78, 17 (2006).

[6] W. S. Bakr, J. I. Gillen, A. Peng, S. Fölling, and M. Greiner, Nature (London) 462, 74 (2009).

[7] J. F. Sherson, C. Weitenberg, M. Endres, M. Cheneau, I. Bloch, and S. Kuhr, Nature (London) 467, 68 (2010).

[8] L. W. Cheuk, M. A. Nichols, M. Okan, T. Gersdorf, V. V. Ramasesh, W. S. Bakr, T. Lompe, and M. W. Zwierlein, Phys. Rev. Lett. 114, 193001 (2015).

[9] E. Haller, J. Hudson, A. Kelly, D. A. Cotta, B. Peaudecerf, G. D. Bruce, and S. Kuhr, Nat. Phys. 11, 738 (2015).

[10] M. F. Parsons, F. Huber, A. Mazurenko, C. S. Chiu, W. Setiawan, K. Wooley-Brown, S. Blatt, and M. Greiner, Phys. Rev. Lett. 114, 213002 (2015).

[11] A. Omran, M. Boll, T. A. Hilker, K. Kleinlein, G. Salomon, I. Bloch, and C. Gross, Phys. Rev. Lett. 115, 263001 (2015).

[12] G. J. A. Edge, R. Anderson, D. Jervis, D. C. McKay, R. Day, S. Trotzky, and J. H. Thywissen, Phys. Rev. A 92, 063406 (2015).

[13] E. Cocchi, L. A. Miller, J. H. Drewes, M. Koschorreck, D. Pertot, F. Brennecke, and M. Köhl, Phys. Rev. Lett. 116, 175301 (2016).

[14] D. Greif, M. F. Parsons, A. Mazurenko, C. S. Chiu, S. Blatt, F. Huber, G. Ji, and M. Greiner, Science 351, 953 (2016).

[15] L. W. Cheuk, M. A. Nichols, K. R. Lawrence, M. Okan, H. Zhang, and M. W. Zwierlein, Phys. Rev. Lett. 116, 235301 (2016).

[16] J. H. Drewes, E. Cocchi, L. A. Miller, C. F. Chan, D. Pertot, F. Brennecke, and M. Köhl, Phys. Rev. Lett. 117, 135301 (2016).

[17] M. F. Parsons, A. Mazurenko, C. S. Chiu, G. Ji, D. Greif, and M. Greiner, Science 353, 1253 (2016). 
[18] M. Boll, T. A. Hilker, G. Salomon, A. Omran, J. Nespolo, L. Pollet, I. Bloch, and C. Gross, Science 353, 1257 (2016).

[19] L. W. Cheuk, M. A. Nichols, K. R. Lawrence, M. Okan, H. Zhang, E. Khatami, N. Trivedi, T. Paiva, M. Rigol, and M. W. Zwierlein, Science 353, 1260 (2016).

[20] J. H. Drewes, L. A. Miller, E. Cocchi, C. F. Chan, N. Wurz, M. Gall, D. Pertot, F. Brennecke, and M. Köhl, Phys. Rev. Lett. 118, 170401 (2017).

[21] A. Mazurenko, C.S. Chiu, G. Ji, M. F. Parsons, M. Kanász-Nagy, R. Schmidt, F. Grusdt, E. Demler, D. Greif, and M. Greiner, Nature (London) 545, 462 (2017).

[22] P. T. Brown, D. Mitra, E. Guardado-Sanchez, P. Schauß, S. S. Kondov, E. Khatami, T. Paiva, N. Trivedi, D. A. Huse, and W. S. Bakr, Science 357, 1385 (2017).

[23] J. Koepsell, J. Vijayan, P. Sompet, F. Grusdt, T. A. Hilker, E. Demler, G. Salomon, I. Bloch, and C. Gross, Nature (London) 572, 358 (2019).

[24] M. A. Nichols, L. W. Cheuk, M. Okan, T. R. Hartke, E. Mendez, T. Senthil, E. Khatami, H. Zhang, and M.W. Zwierlein, Science 363, 383 (2019).

[25] P. T. Brown, D. Mitra, E. Guardado-Sanchez, R. Nourafkan, A. Reymbaut, C.-D. Hébert, S. Bergeron, A.-M. S. Tremblay, J. Kokalj, D. A. Huse, P. Schauß, and W. S. Bakr, Science 363, 379 (2019).

[26] E. Guardado-Sanchez, A. Morningstar, B. M. Spar, P. T. Brown, D. A. Huse, and W. S. Bakr, Phys. Rev. X 10, 011042 (2020).

[27] M. T. DePue, C. McCormick, S. L. Winoto, S. Oliver, and D. S. Weiss, Phys. Rev. Lett. 82, 2262 (1999).

[28] N. Schlosser, G. Reymond, I. Protsenko, and P. Grangier, Nature (London) 411, 1024 (2001).

[29] D. Mitra, P. T. Brown, E. Guardado-Sanchez, S. S. Kondov, T. Devakul, D. A. Huse, P. Schauß, and W. S. Bakr, Nat. Phys. 14, 173 (2018).

[30] Q. Zhou and T.-L. Ho, Phys. Rev. Lett. 106, 225301 (2011).

[31] N. Gemelke, X. Zhang, C.-L. Hung, and C. Chin, Nature (London) 460, 995 (2009).

[32] C. Sanner, E. J. Su, A. Keshet, R. Gommers, Y. I. Shin, W. Huang, and W. Ketterle, Phys. Rev. Lett. 105, 040402 (2010).

[33] T. Müller, B. Zimmermann, J. Meineke, J.-P. Brantut, T. Esslinger, and H. Moritz, Phys. Rev. Lett. 105, 040401 (2010).
[34] E. Cocchi, L. A. Miller, J. H. Drewes, C. F. Chan, D. Pertot, F. Brennecke, and M. Köhl, Phys. Rev. X 7, 031025 (2017).

[35] See Supplemental Material at http://link.aps.org/ supplemental/10.1103/PhysRevLett.125.113601 for more information about the bilayer imaging procedure and additional fluctuation thermometer data.

[36] P. M. Preiss, R. Ma, M. E. Tai, J. Simon, and M. Greiner, Phys. Rev. A 91, 041602(R) (2015).

[37] M. Ku, A. Sommer, L. Cheuk, and M. Zwierlein, Science 335, 563 (2012).

[38] M. W. Zwierlein, in Proceedings of the International School of Physics Enrico Fermi (IOS Press Ebooks, 2016), Vol. 191, pp. 143-220, https://dx.doi.org/10.3254/978-1-61499-694$1-143$.

[39] P. M. Duarte, R. A. Hart, T.-L. Yang, X. Liu, T. Paiva, E. Khatami, R. T. Scalettar, N. Trivedi, and R. G. Hulet, Phys. Rev. Lett. 114, 070403 (2015).

[40] C. N. Varney, C.-R. Lee, Z. J. Bai, S. Chiesa, M. Jarrell, and R. T. Scalettar, Phys. Rev. B 80, 075116 (2009).

[41] C.-H. Cheng and S.-K. Yip, Phys. Rev. B 75, 014526 (2007).

[42] T.-L. Ho and Q. Zhou, Nat. Phys. 6, 131 (2010).

[43] S. Nascimbène, N. Navon, K. J. Jiang, F. Chevy, and C. Salomon, Nature (London) 463, 1057 (2010).

[44] C. Walsh, P. Sémon, G. Sordi, and A.-M. S. Tremblay, Phys. Rev. B 99, 165151 (2019).

[45] A. J. Kim, F. Simkovic, and E. Kozik, Phys. Rev. Lett. 124, 117602 (2020).

[46] H. B. Callen and T. A. Welton, Phys. Rev. 83, 34 (1951).

[47] M. M. Wolf, Phys. Rev. Lett. 96, 010404 (2006).

[48] D. Gioev and I. Klich, Phys. Rev. Lett. 96, 100503 (2006).

[49] B. Swingle, Phys. Rev. Lett. 105, 050502 (2010).

[50] E. Khatami and M. Rigol, Phys. Rev. A 84, 053611 (2011).

[51] M. Endres, M. Cheneau, T. Fukuhara, C. Weitenberg, P. Schauß, C. Gross, L. Mazza, M. C. Bañuls, L. Pollet, I. Bloch, and S. Kuhr, Appl. Phys. B 113, 27 (2013).

[52] N. Bulut, D. J. Scalapino, and R. T. Scalettar, Phys. Rev. B 45, 5577 (1992).

[53] S. Okamoto and T. A. Maier, Phys. Rev. Lett. 101, 156401 (2008).

[54] First results were reported by M. Zwierlein, Quantum transport in strongly interacting Fermi gases, DAMOP 2019, Abstract J06.00003.

[55] J. Koepsell, S. Hirthe, D. Bourgund, P. Sompet, J. Vijayan, G. Salomon, C. Gross, and I. Bloch, Phys. Rev. Lett. 125, 010403 (2020). 